\title{
Quem acessa o Programa Farmácia Popular do Brasil? Aspectos do fornecimento público de medicamentos
}

\author{
The "Farmácia Popular do Brasil" Program and aspects \\ of public provision of medicines in Brazil
}

Cláudia Du Bocage Santos-Pinto ${ }^{1}$

Nilson do Rosário Costa ${ }^{2}$

Claudia Garcia Serpa Osorio-de-Castro ${ }^{1}$

\footnotetext{
${ }^{1}$ Núcleo de Assistência Farmacêutica, Escola Nacional de Saúde Pública Sergio Arouca, Fundação Oswaldo Cruz. Rua Leopoldo Bulhões 1480, sala 632, Manguinhos. 21041-210 Rio de Janeiro RJ.clabocage@fiocruz.br ${ }^{2}$ Departamento de Ciências Sociais, Escola Nacional de Saúde Pública Sergio Arouca, Fundação Oswaldo Cruz.
}

\begin{abstract}
In 2004, the Federal Government introduced the "Farmácia Popular do Brasil" Program, which was an example of policy innovation, establishing a co-payment scheme as a strategy for access to medication. The study analyzed the original model of the Program. Data were obtained from interviews with key stakeholders, program documents and user prescriptions and registers. The results showed widespread expansion of the PFPB network and in the number of people attended. Despite the ever-increasing number of people attended with prescriptions from the private sector, a large demand from public sector users, namely the original focus of the program, was observed. From the standpoint of the federative pact, the program reinstates the centralized model of essential medication distribution which, in the decentralized public system is under state and municipal responsibility. The results point to the difficulty in compliance by states and municipalities with medication distribution responsibilities, mainly in the North and Northeast regions of Brazil. The study concludes that the population has been consistently turning to the PFPB for essential medication it has not been able to access in the public sector.
\end{abstract}

Key words Essential medication, Access, Co-payment, Decentralization, Government programs
Resumo Em 2004, o Governo federal lançou o Programa Farmácia Popular do Brasil, que surge como uma inovação para a política pública de assistência farmacêutica através da adoção do copagamento como estratégia de ampliação do acesso a medicamentos. O trabalho analisou o modelo inicial do Programa, baseado na implantação das unidades de dispensação de medicamentos. Os dados foram obtidos por entrevistas com atores-cha$v e$, análise documental e registros de atendimentos. A análise permitiu identificar a origem da população que utiliza o Programa e descrever o perfil de utilização dos medicamentos dispensados. Os resultados demonstraram grande expansão da rede e do número de atendimentos. Observou-se grande demanda por parte dos usuários do Sistema Único de Saúde (SUS). Do ponto de vista do pacto federativo, o Programa Farmácia Popular do Brasil reedita o modelo de compra centralizada de medicamentos essenciais, que, na lógica do sistema público descentralizado, está sob responsabilidade de provisão dos estados e municípios. As evidências apontam para problemas com a provisão descentralizada, principalmente nas regiões Norte e Nordeste, fazendo com que a população usuária do SUS recorra ao PFPB para provisão dos medicamentos que não logram obter nas unidades públicas de dispensação.

Palavras-chave Medicamentos essenciais, Acesso, Copagamento, Descentralização, Programas governamentais 


\section{Introdução}

No Brasil, o acesso à saúde está inscrito na Constituição como um direito fundamental de todos os cidadãos. Além de direito, é considerado como de dever do Estado, cabendo ao poder político organizar o Sistema Único de Saúde (SUS), formular e implementar ações que assegurem o acesso da população aos serviços de promoção, proteção e recuperação da saúde ${ }^{1}$.

Nesse contexto, em certos segmentos da provisão pública, as demandas sempre crescentes por serviços coexistem com os constrangimentos orçamentários. A proposta de prover tudo para todos inevitavelmente esbarra no problema da escassez. Segundo Elster ${ }^{2}$, a escassez de um bem é dada pela sua insuficiência em atender a todos. Por outro lado, a Assistência Farmacêutica (AF) só se concretiza mediante o acesso aos medicamentos pelos pacientes, nas quantidades adequadas e no momento necessário. Em vista disso, o Estado gestor orienta-se para a provisão de medicamentos considerados essenciais ou que combatam as doenças de grande impacto para a saúde pública ${ }^{3}$.

Entretanto, qualquer planejamento ou política em medicamentos torna-se mais difícil de ser implementado quando pensado para um país como o Brasil, que possui uma população de quase 200 milhões de habitantes e que convive com intensas diferenças inter e intrarregionais. Reduzir essas desigualdades se tornou um desafio para os formuladores das políticas. Uma das estratégias empregadas foi a proposta da descentralização da AF para estados e municípios, iniciada a partir de 1996 com a NOB $96^{4}$ e mais tarde com a Portaria n ${ }^{\circ} 176$, de 8 de março de $1999^{5}$.

Com a descentralização, a responsabilidade pela garantia de acesso a determinados grupos de medicamentos foi dividida entre as esferas de governo. Alguns grupos passaram a ser de responsabilidade do gestor municipal, como os da atenção básica, outros passaram para os gestores estaduais, como os medicamentos de dispensação em caráter excepcional, e outros continuaram sob gerência do governo Federal, como os programas estratégicos ${ }^{3}$.

A descentralização promoveu também o surgimento de um conjunto de normas e diretivas na forma de portarias que tinham como missão organizar o cenário de provisão nas três esferas ${ }^{6}$. No entanto, os constrangimentos de gestão e orçamentários precipitaram o estabelecimento de multiplicidade de regras ao longo do tempo, causando indefinição ao invés de esclarecimento quan- to às responsabilidades de provisão ${ }^{7}$. Mesmo após a redefinição das competências pela Portaria ${ }^{\circ}$ 204, de 29 de janeiro de $2007^{8}$, a situação permanece indefinida em variadas situações, ou tem definição pactuada estado a estado, município a município, o que não ajuda no esclarecimento ao usuário do Sistema Único e Saúde (SUS).

Especula-se, assim, que a descentralização possa não ter sido resolutiva para a questão do acesso aos medicamentos. A descentralização trouxe para os gestores a responsabilidade do ciclo da AF, tarefa esta que envolve atividades de planejamento, organização, coordenação, acompanhamento e avaliação. Mas, aparentemente, não são todos os estados e municípios que vêm logrando executar essas atividades de forma satisfatória. Um fato que viria corroborar esta hipótese seria o crescente número de processos judiciais impetrados pela população contra o Estado, na tentativa de obter medicamentos. Estes se relacionam principalmente aos medicamentos de alto custo, entretanto, entre os processos podem ser encontrados também pedidos de medicamentos essenciais da atenção básica que, teoricamente, deveriam estar sempre disponíveis nas unidades públicas de dispensação ${ }^{7}$.

No entanto, alguns avanços notáveis podem ser destacados, como é o caso da política de combate ao HIV/Aids. Essa política, centralizada quanto à gestão dos recursos, programação e aquisição de medicamentos, gerou uma série de determinantes programáticos que oportunizaram a disponibilização dos antirretrovirais para todos que deles necessitam, sendo modelo de gestão de programa, inclusive em âmbito internacional.

A baixa disponibilidade de medicamentos essenciais nas unidades públicas de saúde penaliza predominantemente os indivíduos mais vulneráveis, os de menor renda, que geralmente dependem da obtenção gratuita de medicamentos pelo setor público como única alternativa de tratamento; além disso, a falta de medicamentos compromete a imagem dos serviços e pode ocasionar internações desnecessárias que oneram o sistema de saúde ainda mais? .

Sabe-se que a compra direta pelos indivíduos representa ainda uma das principais formas de acesso aos medicamentos, situação que se torna mais grave no caso de doenças crônicas que ensejam medicamentos de uso contínuo, muitas vezes com tratamento perdurando por toda a vida do paciente. Dessa forma, devido aos altos custos dos medicamentos e tratamentos, estes podem se tornar inacessíveis para considerável parte da população ${ }^{10}$. Este fato é corroborado 
pelos dados da Pesquisa de Orçamentos Familiares (POF) de 2003, que demonstra o impacto que os medicamentos causam nos orçamentos familiares, podendo chegar a representar 75\% dos gastos com saúde ${ }^{11}$.

No ano de 2004, o Governo federal lançou o Programa Farmácia Popular do Brasil. O Programa surge como estratégia de ampliação do acesso, simultaneamente colocando em questão a efetividade da descentralização da Assistência Farmacêutica, retornando ao modelo de compra centralizada de medicamentos que tem se mostrado eficiente para os Programas sob responsabilidade do Governo federal.

Nessa perspectiva, alguns aspectos que caracterizam o PFPB podem gerar evidências sobre a situação do acesso a medicamentos no setor público. E, mais do que isso, tais evidências podem também caracterizar o Programa como uma nova alternativa à possível baixa efetividade do sistema público de provisão de medicamentos no país.

O objetivo deste trabalho foi elucidar o padrão de implementação do PFPB por meio das unidades próprias, que correspondem à fase inicial do PFPB. A segunda fase, iniciada em 2006, diz respeito à expansão do Programa para a rede privada. Entretanto, esta não foi analisada no estudo devido às diferenças existentes em relação ao modelo da primeira fase. Além disso, buscou-se elucidar os antecedentes, os aspectos estruturais e organizacionais e o padrão de utilização pela população, de modo a confrontar alguns dos resultados do Programa com a política de medicamentos do país.

\section{Estudando o PFPB}

A pesquisa abordou o PFPB, na forma de estudo de caso. Procurou-se descrever o Programa na sua primeira fase, que corresponde à implantação de unidades dispensadoras de medicamentos da Farmácia Popular do Brasil iniciada em 2004, e ainda corrente em todo o país. O estudo não abordou a expansão do Programa para a rede privada, que se deu a partir de 2006.

Foram consultados documentos (leis, portarias e demais documentos envolvidos na criação do PFPB e em seu funcionamento), realizadas entrevistas (atores-chave envolvidos com a criação do Programa) e coletados dados de fontes secundárias do Programa (informações relativas aos atendimentos segundo a origem da po- pulação e o consumo de medicamentos). Os resultados obtidos trouxeram evidências sobre a evolução do Programa, desde sua criação até o final de 2007.

O modelo de investigação proposto utilizou como referência o enfoque da avaliação para a tomada de decisão ou avaliação centrada na administração de programas e políticas ${ }^{12}$. Além disso, utilizou-se como base a metodologia de análise de estrutura, resultados e impactos de intervenções, desenvolvida pela Universidade Estadual de Campinas ${ }^{13}$. Entretanto, o trabalho não se propôs a realizar uma avaliação do PFPB e sim uma descrição de aspectos do Programa que elucidassem sua criação e sua conformação, o que por sua vez proporcionou confrontar os achados com as diretrizes da Política Nacional de Medicamentos.

Os resultados foram analisados por meio de categorias. Estas dizem respeito às unidades (rede), à população, aos medicamentos e aos atendimentos realizados pelo PFPB.

\section{O Programa Farmácia Popular do Brasil}

Nos últimos anos, o debate sobre a reforma dos sistemas nacionais de saúde trouxe à tona a questão do copagamento. Segundo Vianna ${ }^{14}$, esta é uma iniciativa que poderia ser adotada em situações de grandes heterogeneidades, como é o caso do Brasil, entretanto, utilizando-se de alguns critérios seletivos, como o que diz respeito à renda da população, por exemplo.

Iniciativas pontuais de copagamento para acesso a medicamentos ocorreram ao longo dos últimos anos em alguns estados brasileiros. Entretanto, em 2004, o PFPB foi lançado como primeira iniciativa federal de copagamento para acesso a medicamentos, tendo como foco a parcela da população que não utiliza o SUS, mas que, no entanto, não possui rendimentos suficientes para adquirir e/ou completar um tratamento com medicamentos de forma adequada.

A centralização no nível federal proporcionou a padronização das atividades e produtos em todo o país, além dos ganhos de escala decorrentes de grandes compras.

A análise de aspectos relativos à evolução do Programa, tais como unidades, cobertura, consumo de medicamentos e origem da população que acessa o PFPB, permitiu estabelecer uma relação entre o Programa e a atual situação da provisão pública de medicamentos, fruto da descentralização que rege o sistema de saúde. 


\section{PFPB: implantação e expansão 2004-2007}

O Programa surgiu através de uma demanda do Governo federal por uma proposta de ampliação do acesso a medicamentos, que se deu pela constatação da existência, nas grandes metrópoles, de uma grande parcela da população fazendo uso de serviços privados, mas com dificuldade de acesso a medicamentos. A proposta de fornecimento de medicamentos a baixo custo utilizada pelo Laboratório Farmacêutico de Pernambuco (Lafepe) desde $2001^{15}$ passou a servir de modelo para a nova iniciativa do Governo federal. O Programa foi caracterizado pela gestão conjunta entre Ministério da Saúde e Fundação Oswaldo Cruz (Fiocruz), que passou a cumprir o papel de executora do Programa. Isto foi possível por meio da Lei no 10.858 , de 13 de abril de $2004^{16}$, que autoriza a Fundação Oswaldo Cruz a disponibilizar medicamentos mediante ressarcimento, regulamentada pelo Decreto no 5.090, de 20 de maio de $2004^{17}$, que institui o Programa.

O Programa possui três modelos de unidades, sendo o modelo 1 aquele cujas unidades estão sob gestão da Fiocruz; o modelo 2, cujas unidades são estabelecidas através de parcerias da Fiocruz com estados, municípios, órgãos, instituições e entidades sem fins lucrativos; e o modelo 3, estabelecido através de farmácias privadas credenciadas a disponibilizar medicamentos através do Programa, sob gestão direta do MS, sem participação da Fiocruz.

Na prática, as unidades de modelo 1 e modelo 2 são iguais, não havendo diferença para a população. O que as distingue é a gestão, já que as unidades de modelo 1 são geridas pela Fiocruz, sem parcerias, diferente das unidades de modelo 2, que se baseiam na gestão conjunta da Fiocruz com os parceiros. O modelo 3 do Programa, que diz respeito a sua expansão, é gerido diretamente pelo Ministério da Saúde e possui algumas particularidades que o distinguem dos demais, tais como uma lista de medicamentos mais restrita e focada em determinadas condições patológicas; preços de medicamentos que podem variar entre diferentes marcas de um mesmo medicamento ou entre os estabelecimentos participantes do Programa; e um cálculo diferenciado para o valor do subsídio, baseado em um valor de referência estabelecido para cada medicamento. Entretanto, este modelo não foi abordado pelo estudo, uma vez que se estabeleceu de maneira distinta dos modelos da fase inicial do Programa.

A implantação das unidades do PFPB teve início em junho de 2004 com a inauguração de 16 unidades de modelo 1 nos estados da Bahia, do Rio de Janeiro, São Paulo e Goiás. Hoje existem apenas 38 unidades sob gestão exclusiva da Fiocruz, unidades estas que foram inauguradas até agosto de 2005.

Em 2005 deu-se início a inauguração de unidades do modelo 2, que rapidamente passaram a se expandir por outros municípios e estados. Para este modelo foi preestabelecido um limite de 500 unidades, entretanto, em 2007 esta meta foi expandida para 600 unidades. O levantamento de informações sobre as parcerias permitiu perceber o caráter municipal do Programa, já que 92\% das unidades de modelo 2 foram estabelecidas através de demanda dessas instâncias.

A partir de 2006 ocorreu um grande incremento no número de unidades, o maior entre os anos estudados. No final de 2007, o PFPB contava com 407 unidades inauguradas, como pode ser observado no Gráfico 1, deixando nítida a grande evolução ocorrida desde 2004.

No que diz respeito aos estados contemplados, foi evidenciada a implantação de maior número de unidades nos estados das regiões $\mathrm{Su}$ deste (SE) e Nordeste (NE), em todo o período estudado. No entanto, ao longo do tempo, houve incremento importante nas regiões Norte $(\mathrm{N})$ $(817 \%)$ e Sul (S) (860\%), como pode ser observado na Tabela 1 . Com relação aos municípios, foi observada a presença de mais de uma unidade do PFPB em alguns municípios, principalmente nas regiões SE e NE, o que caracteriza também um padrão diferente de concentração municipal do Programa nestas regiões.

De acordo com o Programa, para a adesão ao PFPB a localidade candidata deveria se enquadrar em critérios estabelecidos. Segundo suas diretrizes, as unidades deveriam ser instaladas, prioritariamente, em locais estratégicos, municípios em que o perfil socioeconômico da população fosse compatível com os objetivos do Programa, com população que utiliza o setor privado e sobre a qual os custos do tratamento medicamentoso incidem de forma mais importante. Além disso, estabeleceu-se que os municípios candidatos a receber unidades do Programa deveriam atender ao critério de possuírem mais de 100.000 habitantes, no estado de São Paulo, e mais de 70.000 habitantes nos demais estados do país.

Uma vez atendidas as regiões estratégicas, foi dada alternativa para aqueles municípios que não atingiam tais critérios populacionais. Estes poderiam possuir unidades que atendessem a uma microrregião, levando-se em consideração a importância regional, a área abrangida e a acessibilidade. 


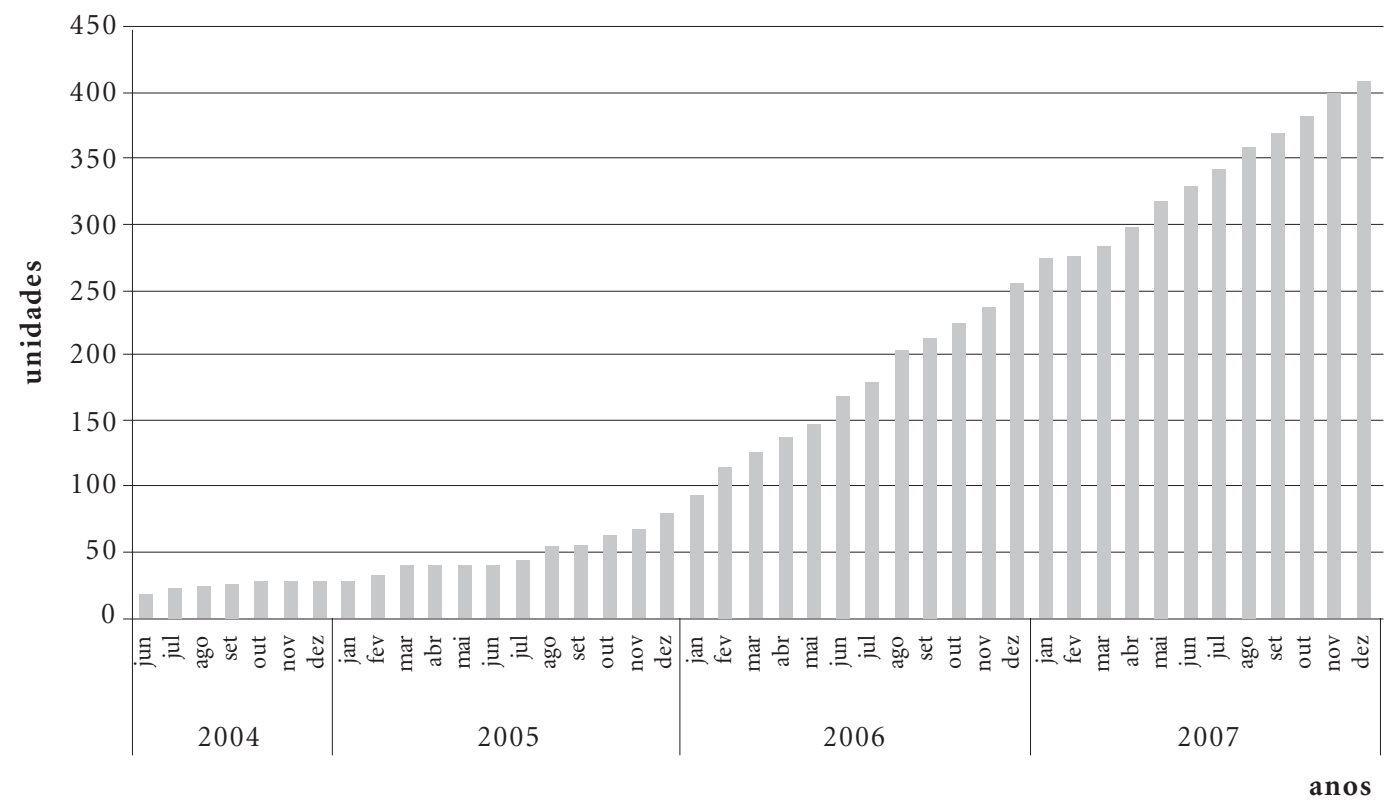

Gráfico 1. Evolução das unidades do PFPB entre os anos de 2004 e 2007 - PFPB, 2007.

Tabela 1. Incremento de unidades do PFPB entre os anos de 2005 e 2007 por região - PFPB, 2007.

\begin{tabular}{lrrrrr}
\hline & \multicolumn{2}{c}{ Anos } & & \multicolumn{2}{c}{ Incremento } \\
\cline { 2 - 3 } \cline { 5 - 6 } Regiões & $\mathbf{2 0 0 5}$ & $\mathbf{2 0 0 7}$ & & unidades & percentual \\
\hline $\mathrm{N}$ & 6 & 55 & & 49 & 817 \\
$\mathrm{NE}$ & 22 & 137 & & 115 & 523 \\
$\mathrm{SE}$ & 41 & 146 & & 105 & 256 \\
$\mathrm{~S}$ & 5 & 48 & & 43 & 860 \\
$\mathrm{CO}$ & 4 & 20 & & 16 & 400
\end{tabular}

Os resultados da análise sugerem que a maioria dos municípios contemplados com unidades do Programa obedeceu aos pré-requisitos populacionais. Mas foram observados casos em que os municípios contemplados possuíam um número menor de habitantes do que o estipulado como mínimo pelo Programa, com destaque para os estados de Mato Grosso do Sul, Paraíba e Rio Grande do Sul, onde a proporção de municípios com menos de 70.000 habitantes superou a proporção de municípios com mais de 70.000 habitantes. Entretanto, a maioria desses municípios contemplava uma microrregião, por isso enquadravam-se nos critérios estabelecidos. Foram observados, ainda, 19 municípios atendidos pelo
Programa que não se enquadravam em nenhum dos critérios, sendo 7 em Minas Gerais, 3 no Rio Grande do Sul, 2 na Bahia, 2 no Pará, 1 em Santa Catarina, 1 no Amazonas, 1 no Espírito Santo, 1 no Mato Grosso do Sul e 1 no Rio de Janeiro.

Cada unidade possui ainda uma respectiva população de abrangência. A depender do município onde a unidade está instalada, essa população pode ou não corresponder ao número total de habitantes, mas sempre irá relacionar-se à população que, segundo dados do próprio município, tem efetiva possibilidade de utilizar a unidade. Nesse caso, os resultados da análise conduzida neste trabalho explicitam que a população total abrangida pelas unidades do Programa, no final de 2007, chegava a mais de 92 milhões de pessoas. As regiões SE e NE demonstraram que suas unidades abrangiam cerca de 40 milhões e 25 milhões de pessoas, respectivamente, enquanto as demais regiões não ultrapassaram 10 milhões.

Tanto SE quanto NE são as regiões que possuem maior densidade populacional. Assim, a abrangência poderia ser explicada, tanto pelo quantitativo de unidades instaladas como pela maior concentração populacional nessas regiões.

Apesar de a análise em números absolutos ter demonstrado a região SE como a de maior 
abrangência populacional, quando comparamos a população abrangida pelo Programa com a população total de cada região percebe-se que as regiões $\mathrm{N}$ e NE são aquelas em que, proporcionalmente, a população tem maior possibilidade de utilizar o Programa. A população abrangida nessas regiões representa mais de $50 \%$ da população total.

No entanto, quando se compara a população abrangida com a média da população atendida entre 2005 e 2007 (Tabela 2), observa-se que a média de atendimentos nas regiões foi de 5,7\% da população abrangida, sendo a Região Centro-Oeste aquela cuja população mais utiliza o Programa (7,6\%).

Por não se ter estabelecido um padrão quanto a taxas mínimas de utilização em Programas desse tipo, torna-se difícil avaliar se a utilização efetiva do PFPB é realmente baixa.

\section{Provimento dos medicamentos selecionados}

A lista de medicamentos do Programa é composta por 107 itens, mas este número se reduz para 97 (acrescido do preservativo masculino) caso não se considerem as variações de tamanho de embalagem, o que acontece para 10 itens. Deste modo, existem 96 apresentações, representando 71 medicamentos de diferentes classes terapêuticas. Deste número total de apresentações, 76 constam na Relação Nacional de Medicamentos (Rename) de 2006. Dos 20 restantes, 12 constavam na Rename 2002, mas foram retirados ou substituídos na nova edição; 17 estão presentes na Rename 2006, mas em concentrações ou formas farmacêuticas distintas. Oito medicamentos do PFPB não constam em nenhuma das duas últimas Renames.

A lista contempla um grande número de classes terapêuticas; entretanto, algumas estão menos representadas. Outras, como os anti-infectantes $(25 \%)$, medicamentos que atuam sobre o sistema nervoso central e periférico (12\%) e me- dicamentos que atuam sobre o sistema cardiovascular e renal (12\%), estão mais representadas e juntas representam praticamente $50 \%$ da lista de medicamentos do Programa.

A análise do consumo dos medicamentos no PFPB demonstrou que, em todas as regiões, são praticamente os mesmos medicamentos que se encontram entre os mais consumidos: ácido acetilsalicílico, atenolol, captopril, enalapril, hidroclorotiazida, metformina, nifedipina, omeprazol, ranitidina, sinvastatina e paracetamol, todos considerados essenciais pela lista oficial brasileira ${ }^{18}$. Dos 11 medicamentos citados entre os 10 mais vendidos, em todas as regiões, $64 \%$ são medicamentos que atuam sobre o sistema cardiovascular.

Verificou-se ainda que mais de 70\% dos medicamentos do PFPB também fazem parte do elenco de Programas do MS, o que não é surpreendente, visto que ambos se baseiam na Rename e acabam se voltando para as condições de saúde mais prevalentes entre a população. Tal fato demonstra que o Programa é uma opção para a obtenção de medicamentos tanto para usuários da rede privada como para os usuários da rede pública.

\section{População que acessa o Programa e efetividade da implementação do PFPB}

A criação do Programa teve como objetivo principal atingir a faixa populacional com orçamento familiar entre 4 e 10 salários mínimos que utiliza o sistema de saúde privado. Entretanto, o PFPB não estabelece barreiras de acesso à população, a não ser a prescrição do medicamento, seja ela da rede pública ou privada.

O Programa chama a recepção do usuário, seguido de verificação da receita e seu aviamento, de atendimento. Este atendimento nem sempre inclui orientação; esta é realizada mediante solicitação do usuário. A análise dos atendimentos realizados pelo Programa, entre os anos de 2005 e 2007, mostrou que, de acordo com os objetivos do $\mathrm{PFPB}$, a maioria deles referiu-se à população

Tabela 2. Proporção entre a média de atendimentos realizados pelo PFPB, em todos os seus anos, e a população abrangida por região - PFPB, 2007.

\begin{tabular}{lrrrrrr}
\hline & \multicolumn{1}{c}{ N } & \multicolumn{1}{c}{ NE } & \multicolumn{1}{c}{ SE } & \multicolumn{1}{c}{ S } & \multicolumn{1}{c}{ CO } & \multicolumn{1}{c}{ Brasil } \\
\hline $\begin{array}{l}\text { População abrangida } \\
\text { Média da população atendida }\end{array}$ & 8.667 .219 & 28.395 .683 & 41.866 .865 & 10.121 .637 & 3.827 .203 & 92.878 .607 \\
entre os anos de 2004 e 2007 & 505.279 & 1.383 .026 & 2.714 .924 & 461.750 & 292.936 & 5.357 .915 \\
\begin{tabular}{l} 
Proporção \\
\hline
\end{tabular} & $5,83 \%$ & $4,87 \%$ & $6,48 \%$ & $4,56 \%$ & $7,65 \%$ & $5,77 \%$ \\
\hline
\end{tabular}


não proveniente da rede pública de saúde, como pode ser observado no Gráfico 2. Pode-se perceber que, em todos os anos, tanto o número de atendimentos a usuários do setor público quanto a usuários do setor privado vêm crescendo, ainda que a diferença entre o número de usuários desses setores pareça diminuir em 2007.

A Tabela 3 corrobora essa informação, demonstrando que, em todos os anos do Programa, a maioria dos atendimentos realizados dizia respeito à população proveniente do setor privado. Entretanto, a análise isolada demonstra que o perfil é diferenciado entre regiões. Na região N, em todos os anos estudados, o número de aten- dimentos à população proveniente da rede pública suplantou o da rede privada. A mesma situação pode ser observada no ano de 2007 para a Região NE, sendo que nos demais anos a proporção foi quase de 50\%. A análise permitiu observar também que, em alguns estados, o número de atendimentos se refere, quase que exclusivamente, à população originária do setor público, como é o caso de Amazonas, Acre e Amapá, na Região N, ou Maranhão, na Região NE, os quais, em todos os anos de existência do Programa, apresentaram mais de $70 \%$ de seus atendimentos à população provenientes da rede pública de saúde.
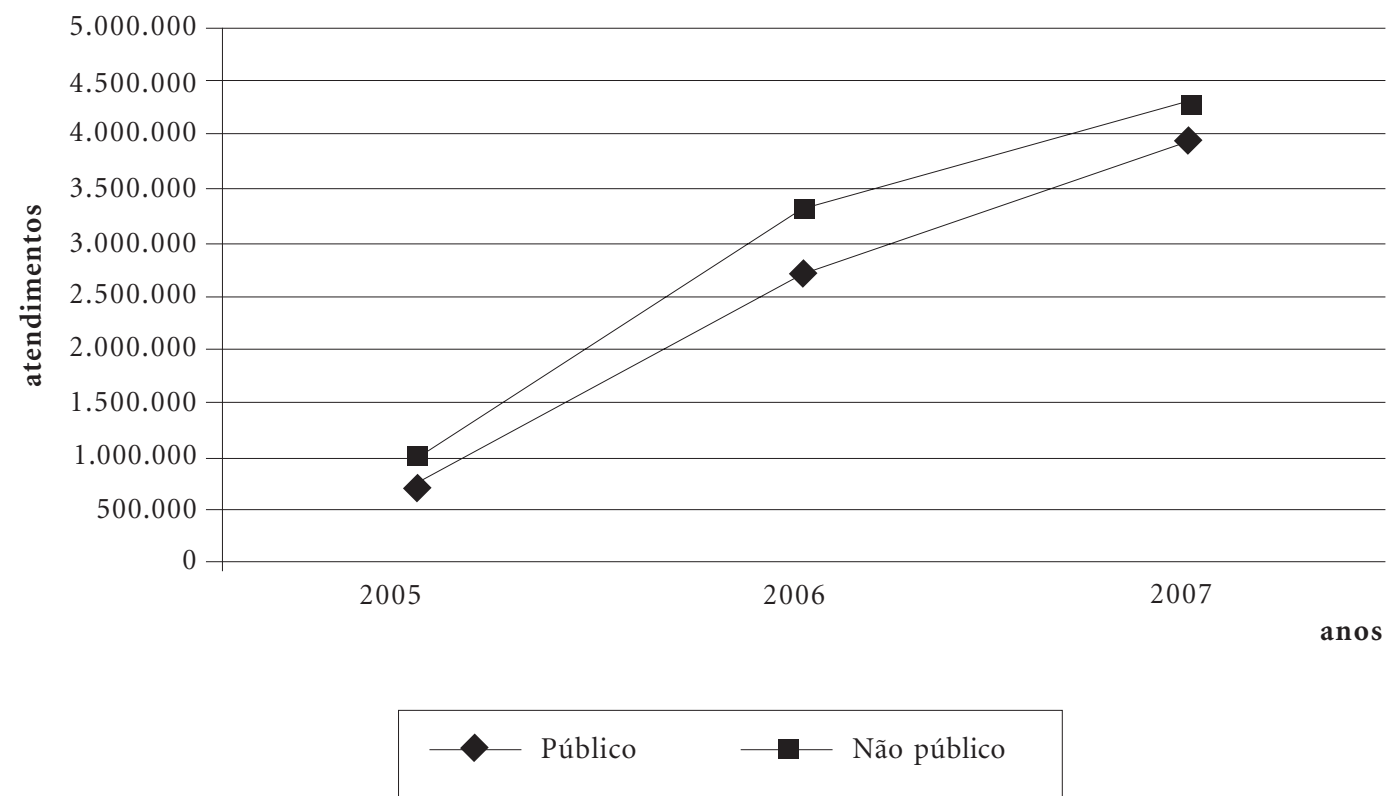

Gráfico 2. Evolução dos atendimentos realizados pelo Programa ao longo dos anos, de acordo com a origem da população - PFPB, 2007.

Tabela 3. Atendimentos realizados pelo PFPB, por anos, segundo o setor - PFPB, 2007.

\begin{tabular}{|c|c|c|c|c|c|c|}
\hline \multirow[b]{2}{*}{ Regiões } & \multicolumn{2}{|c|}{2005} & \multicolumn{2}{|c|}{2006} & \multicolumn{2}{|c|}{2007} \\
\hline & Público & Não público & Público & Não público & Público & Não público \\
\hline $\mathrm{N}$ & 106.010 & 55.369 & 391.500 & 196.309 & 475.315 & 288.895 \\
\hline $\mathrm{NE}$ & 233.042 & 244.823 & 708.618 & 745.999 & 1.231 .542 & 985.054 \\
\hline SE & 352.824 & 515.536 & 1.344 .808 & 1.895 .755 & 1.729 .110 & 2.306 .738 \\
\hline S & 28.818 & 69.319 & 164.754 & 307.481 & 319.607 & 495.270 \\
\hline $\mathrm{CO}$ & 37.996 & 122.978 & 115.990 & 176.405 & 197.700 & 230.179 \\
\hline Total & 758.690 & 1.008 .025 & 2.725 .670 & 3.321 .949 & 3.953 .274 & 4.306 .136 \\
\hline
\end{tabular}


O contrário ocorre nas demais regiões, onde a maior parcela dos atendimentos se deu para população proveniente de serviços de saúde privados. A Região SE manteve praticamente constante a proporção de atendimentos à população proveniente de ambos os setores, em todos os anos, com maioria dos atendimentos $(58 \% \mathrm{em}$ média) para usuários da rede privada. Tanto na Região S como na Região CO, o número de atendimentos à população proveniente do setor público veio crescendo mais do que o de atendimentos a usuários do setor privado, demonstrando uma tendência de inversão dessa proporção. Assim, pode-se observar que o objetivo do PFPB de atender à população proveniente do setor privado esteve mais próximo nas regiões $\mathrm{SE}$, $\mathrm{S}$ e $\mathrm{CO}$, mas não nas regiões $\mathrm{NE}$ e $\mathrm{N}$.

\section{$O$ que os dados mostram sobre a efetividade do PFPB}

Através das análises foi possível acompanhar a evolução do PFPB através de parâmetros como a rede instalada, população atingida, medicamentos consumidos e atendimentos realizados. Em 2007, o PFPB atingiu 407 unidades em todos os estados do país. Foi possível perceber uma grande concentração do Programa em municípios das regiões NE e SE, no entanto, a população abrangida pelo Programa foi proporcionalmente maior nas regiões $\mathrm{N}$ e $\mathrm{NE}$.

Entende-se que a população abrangida deva estar relacionada com o público-alvo do Programa, mesmo que usuários do setor público também acabem adquirindo medicamentos nas unidades do PFPB. No entanto, é de certo modo paradoxal o fato de que $\mathrm{N}$ e $\mathrm{NE}$, as regiões de mais baixo poder aquisitivo do país ${ }^{19}$, apresentem as maiores populações abrangidas. A intenção explicitada pelo Programa é de cobrir população de classe média baixa com alguma possibilidade de pagar pelos medicamentos. A intenção não parece ser coerente com este achado, o que remete à reflexão sobre o real papel que o Programa pode estar desempenhando em alguns locais e quais as motivações para alguns municípios pleitearem a implantação de unidades do PFPB. Será esta tendência resultado da percepção dos próprios gestores locais de que o PFPB viria como alternativa à ineficiência da provisão gratuita?

Com relação aos atendimentos, foram registrados, até dezembro de 2007, mais de 16 milhões em todo o Brasil. As regiões Sudeste e Nordeste foram as que realizaram mais atendimen- tos entre todas as demais, em todos os anos do Programa. Além disso, a demanda por medicamentos, em todas as regiões, demonstrou um perfil de consumo muito similar, com prevalência de medicamentos relacionados a condições cardiovasculares.

A análise da procedência da população atendida revelou que, em sua maioria, são originários do setor privado. Entretanto, a análise de cada região, separadamente, demonstrou que este fato não reflete a realidade de todas, já que as regiões Norte (em todos os seus anos) e Nordeste (em 2007) demonstraram maior número de atendimentos à população proveniente da rede pública. Já as demais regiões seguiram o perfil demonstrado pelo dado agregado, entretanto todas vêm demonstrando, ao longo dos anos, crescimento na proporção de atendimentos a usuários do SUS. Qual seria o motivo desse comportamento? Seria consequência de falhas na provisão pública de medicamentos?

Esta é uma hipótese que deve ser levada em consideração, principalmente ao se observar o dado global e perceber que, independentemente de haver maior número de atendimentos a usuários da rede privada, o número de atendimentos à usuários da rede pública é muito grande, mais de 7 milhões, representando 46\% dos atendimentos realizados pelo Programa.

É claro que tal situação não se deve, única e exclusivamente, a um fator isolado. A literatura sobre o copagamento traz a informação de que muitos usuários não se importam em pagar pelo serviço ou pelo medicamento desde que tenham acesso a eles ${ }^{20}$. Além disso, fatores como a distância até a unidade de saúde, tempo de espera pelo medicamento ou atendimento, contrastando com o bom atendimento nas unidades do PFPB e a pronta disponibilidade do medicamento, também podem influir na opção pelo Programa.

Entretanto, todos são fatores relacionados ao acesso, e caso algum deles esteja comprometido, todo o acesso, através da rede pública, também estará. Desta forma, o número de atendimentos a usuários provenientes do SUS reflete problemas com a provisão pública, o que faz com que seus usuários recorram às unidades do PFPB em busca do medicamento que deveria ter obtido na unidade de saúde.

\section{O PFPB ante a PNM e a descentralização}

Estudo realizado por Ferreira ${ }^{21}$ apresentou dados de satisfação do PFPB. Segundo seus usuários, este se destaca por conseguir prover medi- 
camentos à população de forma satisfatória ${ }^{21}$. Esta situação poderia estar relacionada com o provimento contínuo de medicamentos, característica de boa gestão que, no caso do PFPB, é centralizada, e padroniza o atendimento às condições de saúde prevalentes entre a população, com medicamentos, em sua maioria, essenciais.

O Programa inaugura a convivência de dois sistemas situados em uma mesma gestão. Entretanto, possuem diferenças marcantes, trazendo à tona, mais uma vez, o embate entre a proposta descentralizada e a centralizadora na provisão de medicamentos. Não se pode dizer que com a criação do Programa o Governo federal retoma a opção pela gestão centralizada do sistema; entretanto, também não se pode negar a influência dess modelo na concepção do Programa. Tal característica reforça o papel, mesmo que não intencional, do PFPB como indicador de falhas na provisão pública de medicamentos no país.

$\mathrm{O}$ antigo modelo de gestão de provisão de medicamentos vem de experiências anteriores como a Ceme ${ }^{22}$ e o Programa Farmácia Básica ${ }^{23}$. Atualmente, este modelo ainda é utilizado para a provisão de medicamentos específicos como os antirretrovirais e de demais programas estratégicos, geridos pelo nível federal com a compra e a gestão centralizadas ${ }^{24}$.

Uma vez que o desabastecimento não pode ser admitido para qualquer grupo de medicamentos pactuados pelos três níveis de gestão, por que existiria tratamento diferenciado para alguns? Em relação às atuais iniciativas de gestão centralizada, entre as quais o PFPB, seriam estas resultado da avaliação pelo Governo federal de falhas na provisão descentralizada da AF? Como se partilharia então essa decisão no nível das Comissões Gestoras do SUS?

O PFPB utiliza também o aprendizado gerado pela experiência descentralizadora do sistema, já que é a autonomia dos parceiros, em sua maioria municípios, que determina a participação no Programa. Entretanto, em momento algum deixa de ser estreitamente controlado e regido pelo Executivo federal.

Na política descentralizada do SUS, as ações de provisão de serviços são difusas e nem sempre exitosas. As responsabilidades de cada nível federativo de governo são inespecíficas; com isso, muitas vezes, para a população, a percepção é de que o sistema como um todo é ineficiente. A descentralização, portanto, torna mais difícil para o
Executivo federal retirar para si o reconhecimento pela iniciativa política, fruto principalmente da percepção positiva dos usuários de um programa $^{23}$. O PFPB, como marca de governo, parece resgatar a possibilidade de associação do Executivo federal à política de provisão de bens de saúde. O PFPB centraliza a relação do Ministério da Saúde com a provisão dos medicamentos essenciais.

A partir dessa relação, o Programa, mesmo que de forma não intencional, passa a funcionar como um marcador da outra forma de provisão (provisão pública descentralizada). O elenco de medicamentos possibilita estabelecer tal relação, já que os distintos tipos de usuários utilizam os mesmos medicamentos. Os resultados demonstram que a grande procura do Programa por usuários da rede pública sinaliza ineficiências na provisão de medicamentos pelo SUS em alguns municípios, o que poderia ser atribuído, entre outros fatores, à má gestão do ciclo da assistência farmacêutica neles. Além disso, episódios narrados na imprensa ratificam esses achados, indicando que, em alguns municípios, o PFPB vem suprindo a demanda do serviço público devido às constantes faltas de medicamentos nas unidades ${ }^{25}$.

Caso seja essa a questão, seria importante verificar se os municípios que aderem ao Programa têm seu plano de Assistência Farmacêutica em funcionamento. Os municípios que possuem e cumprem seu Plano deveriam ser aqueles prioritários na adesão ao Programa, uma vez que assim estaria assegurado o direcionamento do Programa à população-alvo e não à população SUS.

$\mathrm{O}$ estudo apresentou algumas limitações. Uma delas se deve à falta de dados sobre as questões financeiras do Programa, o que seria interessante examinar para avaliar o impacto sobre os gastos públicos. Além disso, existe a possibilidade de viés de informação, já que as estatísticas de atendimento são informadas pelas próprias unidades. Por fim, cabe reiterar que a análise só levou em consideração a primeira fase do Programa, pela percepção de maior investimento do PFPB, por sua estrutura formal mais bem definida e por sua ligação direta com a Fiocruz. Entretanto, uma análise da segunda etapa, referente à expansão do Programa para a rede privada, poderia trazer novos resultados e um panorama mais abrangente sobre o acesso a medicamentos por meio de copagamento no país, em vista da maior capilaridade alcançada por esta modalidade do PFPB. 


\section{Conclusão}

Não é possível afirmar as causas da busca por medicamentos no PFPB. Existem hipóteses positivas como a presença contínua de medicamentos nas unidades ou o bom atendimento oferecido; e as negativas, para o SUS, como a falta de medicamentos ou a insatisfação com o atendimento na rede pública. Mas o fato é que muitos usuários que deveriam estar obtendo seus medicamentos de forma gratuita vêm optando por obtê-los mediante desembolso direto.

$\mathrm{O}$ estudo mostrou que, no exame do desenvolvimento do PFPB na sua face de articulação com parceiros públicos, puderam-se evidenciar vulnerabilidades do sistema de provisão de medicamentos no Brasil que ainda não podiam ser avaliados.

A incapacidade do SUS em atender a toda a população abriu espaço para a atuação da assistência médica suplementar a partir da década de 90. Da mesma forma, o PFPB vem absorvendo uma grande proporção de usuários provenientes do SUS, já que o cenário demonstrado sugere que o setor público não vem sendo capaz de prover medicamentos, de forma suficiente, à população.

A descentralização da saúde trouxe resultados de ampliação de acesso importantes, entretanto não tem se mostrado eficaz para a gestão da assistência farmacêutica em todos os municípios do Brasil. O comportamento diferenciado das regiões Norte e Nordeste pode ser explicado pela capacidade diferenciada de provisão dos municípios e estados, que atendem de forma distinta sua população.

Seguindo os moldes de outros "pacotes de atividades" prestadas pelo município, o PFPB possibilita o repasse de recursos fundo a fundo aos municípios credenciados ao Programa. Este talvez seja um dos grandes incentivos à sua expansão. Além disso, aqueles municípios que não cumprem com as funções básicas de provisão de medicamentos podem estar vendo no Programa uma possibilidade extra para solução desse problema, pelo fato de as unidades instaladas serem vinculadas a um recurso novo, independentemente da receita municipal para a saúde. Esse processo lança enorme dúvida sobre a institucionalidade da política pública de assistência farmacêutica do país.

Cabe lembrar que o Programa funciona de forma independente, e sua criação não alterou as responsabilidades de estados e municípios na provisão de medicamentos à população. Assim, o PFPB, junto à judicialização e ao Projeto de Lei no $219 / 07^{26}$, caracteriza o cenário atual de problemas no acesso a medicamentos através do SUS.

\section{Colaboradores}

CDB Santos-Pinto trabalhou na pesquisa, na análise e na redação do artigo; NR Costa e CG Osorio-de-Castro trabalharam no formato final do texto e orientaram a dissertação que deu origem ao artigo.

\section{Agradecimentos}

A Hayne Felipe da Silva, pelo apoio na realização do trabalho e no acesso aos dados, e a Reinaldo Guimarães, pela autorização para a sua utilização. 


\section{Referências}

1. Brasil. Constituição da República Federativa do Brasil 1988. Brasília: Senado Federal; 1988.

2. Elster J. Local Justice. New York: Russell Sage Foundation, 1992.

3. Brasil. Portaria GM no 3.916 , de 30 de outubro de 1998. Dispõe sobre a aprovação da Política Nacional de Medicamentos. Ministério da Saúde. Diário Oficial da União 1998; 18 nov.

4. Brasil. Portaria GM/MS no 2.203, de 5 de novembro de 1996. Aprova a NOB-SUS 01/96. Diário Oficial da União 1996; 6 jul.

5. Brasil. Portaria GM/MS no 176 , de 8 de março de 1999. Estabelece critérios e requisitos para a qualificação dos municípios e estados ao incentivo à Assistência Farmacêutica Básica e define valores a serem transferidos. Brasília: Ministério da Saúde. Diário Oficial da União 1999; 11 mar.

6. Levcovitz E, Lima LD, Machado CV. Política de saúde nos anos 90: relações intergovernamentais e o papel das Normas Operacionais Básicas. Cien Saúde Colet 2001; 6(2): 269-291.

7. Messeder AM, Osório-de-Castro CGS, Luiza VL. Mandados judiciais como ferramenta para garantia do acesso a medicamentos no setor público: a experiência do Estado do Rio de Janeiro. Cad Saude Publica 2005; 21(2):525-534.

8. Brasil. Portaria GM n ${ }^{\circ} 204$, de 29 de janeiro de 2007. Regulamenta o financiamento e a transferência dos recursos federais para as ações e os serviços de saúde, na forma de blocos de financiamento, com o respectivo monitoramento e controle. Brasília, Ministério da Saúde. Diário Oficial da União 2007; 31 jan.

9. Guerra AA, Assis Acúrcio F, Gomes CAP, Miralles M, Werneck GAF, Carvalho CL. Disponibilidade de medicamentos essenciais em duas regiões de Minas Gerais, Brasil. Rev Panam Salud Publica 2004; 15(3):168-175.

10. Luiza VL. Acesso a medicamentos essenciais no Estado do Rio de Janeiro [tese]. Rio de Janeiro: Escola Nacional de Saúde Pública Sergio Arouca, Fundação Oswaldo Cruz; 2003.

11. Instituto Brasileiro de Geografia e Estatística (IBGE). $\backslash$ Pesquisa de Orçamentos Familiares (POF) 2003. [acessado 2007 maio 2005]. Disponível em: http://www. ibge.gov.br/home/estatistica/populacao/condicao devida/pof/2003/default.shtm

12. Worthen BR, Sanders JR, Fitzpatrick J. Avaliação de programas: concepções e práticas. São Paulo: Gente; 2004.

13. Núcleo de Estudos de Políticas Públicas da Universidade Estadual de Campinas (NEPP). Universidade Estadual de Campinas (Unicamp). Banco Interamericano de Desenvolvimento (BID). Efetividade do desenvolvimento em saúde: experiências exitosas na América Latina e no Caribe. Campinas: NEPP, Unicamp, BID; 2006.

14. Vianna SM, Piola SF, Reis COO. Gratuidade no SUS: controvérsias em torno do co-pagamento. Brasília: Instituto de Pesquisa Econômica Aplicada (Ipea); 1998. (Série Texto para Discussão, no 587).

15. Laboratório Farmacêutico do Estado de Pernambuco (LAFEPE). Farmácias LAFEPE. [acessado 2006 jun.6]. Disponível em: http://www.lafepe.pe.gov.br/ farmacias
16. Brasil. Lei no 10.858 , de 13 de abril de 2004. Autoriza a Fundação Oswaldo Cruz-Fiocruz a disponibilizar medicamentos, mediante ressarcimento, e dá outras providências. Diário Oficial da União 2004; 13 abr.

17. Brasil. Decreto no 5.090 , de 20 de maio de 2004 Regulamenta a Lei $\mathrm{n}^{\circ} 10.858$, de 13 de abril de 2004, e institui o programa "Farmácia Popular do Brasil", e dá outras providências. Diário Oficial da União 2004; 21 maio.

18. Brasil. Ministério da Saúde. Rename 2006: processo de revisão e atualização. Brasília: Ministério da Saúde; 2006.

19. Instituto Brasileiro de Geografia e Estatística (IBGE). Produto Interno Bruto dos Municípios 2002-2005. Rio de Janeiro: IBGE; 2007. [acessado 2008 fev 2]. Disponível em: http://www.ibge.gov.br/home/estatistica/ economia/pibmunicipios/2005/tab01.pdf

20. Knippenberg R, Alihonou E, Soucat A, Oyegbite K, Calivis M, Hopwood I, Niimi R, Diallo MP, Conde $\mathrm{M}$, Ofosu-Amaah S. Implementation of the Bamako Initiative: strategies in Benin and Guinea. Int $J$ Health Plann and Manag 1997; 12(Suppl.1):29-47.

21. Ferreira RL. Análise de implantação do Programa Farmácia Popular do Brasil no Rio de Janeiro e no Distrito Federal: um estudo de casos [dissertação]. Rio de Janeiro: Escola Nacional de Saúde Pública Sergio Arouca, Fundação Oswaldo Cruz; 2006.

22. Presidência da República. Central de Medicamentos. Plano Diretor de Medicamentos, 1a etapa: 1973/ 1979, Plano de Ação. Brasília: Presidência da República; 1973.

23. Cosendey MAE. Análise da implantação do Programa Farmácia Básica: um estudo multicêntrico em cinco estados do Brasil [tese]. Rio de Janeiro: Escola Nacional de Saúde Pública Sergio Arouca, Fundação Oswaldo Cruz; 2000.

24. Brasil. Portaria GM n ${ }^{\circ}$ 204, de 29 de janeiro de 2007. Regulamenta o financiamento e a transferência dos recursos federais para as ações e os serviços de saúde, na forma de blocos de financiamento, com o respectivo monitoramento e controle. Brasília, Ministério da Saúde. Diário Oficial da União 2007; 31 jan.

25. Faltam medicamentos na farmácia municipal. Jornal de Araxá. [acessado 2008 fev. 29]. Disponível em: http://www.jornalaraxa.com.br:80/jornalTemp/ noticias.asp?cod_materia $=847 \& \$$ pub $=$ saude

26. Brasil. Projeto de Lei do Senado $n^{\circ} 219$, de 2007. Altera a Lei $n^{\circ}$ 8.080, de 19 de setembro de 1990, para dispor sobre a oferta de procedimentos terapêuticos e a dispensação de medicamentos pelo Sistema Único de Saúde (SUS). Brasília: Senado Federal; 2007.

Artigo apresentado em 21/06/2008

Aprovado em 12/10/2008

Versão final apresentada em 20/11/2008 